Article

\title{
Arrhythmia ECG Noise Reduction by Ensemble Empirical Mode Decomposition
}

\section{Kang-Ming Chang}

Department of Photonics and Communication Engineering, Asia University, Wufeng, Taichung County, 500, Lioufeng Rd., Wufeng, Taichung County, 41354, Taiwan

* Author to whom correspondence should be addressed; E-Mail: changkm@ asia.edu.tw; Tel.: +886-4-23323456; Fax: +886-4-23316699.

Received: 29 April 2010; in revised form: 20 May 2010 / Accepted: 10 June 2010 / Published: 17 June 2010

\begin{abstract}
A novel noise filtering algorithm based on ensemble empirical mode decomposition (EEMD) is proposed to remove artifacts in electrocardiogram (ECG) traces. Three noise patterns with different power-50 Hz, EMG, and base line wander - were embedded into simulated and real ECG signals. Traditional IIR filter, Wiener filter, empirical mode decomposition (EMD) and EEMD were used to compare filtering performance. Mean square error between clean and filtered ECGs was used as filtering performance indexes. Results showed that high noise reduction is the major advantage of the EEMD based filter, especially on arrhythmia ECGs.
\end{abstract}

Keywords: arrhythmia ECG; ensemble empirical mode decomposition; composite noise; filter

\section{Introduction}

Empirical mode decomposition (EMD) is a novel recently developed algorithm [1]. EMD is based on a decomposition derived from the data and is useful for the analysis of nonlinear and nonstationary time series signals [2]. With iterative decomposition of signals, EMD separates the full signal into ordered elements with frequencies ranged from higher to lower frequencies in each intrinsic mode function (IMF) level. Different from the classical Fourier decomposition with sine and cosine basis functions, EMD depends on the characteristics of the signal; therefore EMD behaves as a filter bank 
without a predefined cut-off frequency [2]. This interesting property of EMD has been widely applied in biomedical signal analysis, such as monitoring the effect of anesthetic drugs [3], rapid screening of obstructive sleep apnea [4], and respiratory sinus arrhythmia estimation from ECGs [5].

EMD is also used for ECG noise reduction [6-9]. Blanco-Velasco developed an EMD-based algorithm to remove the baseline wander and high-frequency noise of ECGs [10]. Nimunkar and Tompkin added a pseudo-high-frequency noise to IMFs as an aid to remove power-line noise. They also developed a complete ECG processing algorithm for R-peak detection and feature extraction, based on EMD approaches [11]. Owing to the fact that the lower IMF levels correspond to higher frequency components and vice versa, reconstruction without the lower IMF level can remove highfrequency noise. Thus, low-frequency baseline wander can be removed by reconstruction without higher IMF levels [12].

The major disadvantage of EMD is the so-called mode mixing effect. Mode mixing indicates that oscillations of different time scales coexist in a given IMF, or that oscillations with the same time scale have been assigned to different IMFs. Hence, ensemble EMD (EEMD) was introduced to remove the mode-mixing effect [13]. The principle of the EEMD is to add white noise into the signal with many trials. The noise in each trial is different, and the added noise can be canceled out on average, if the number of trials is sufficient. Thus, as more and more trials are added to the ensemble, the residual part is the signal. EEMD was also widely used for signal processing. For example, reconstruction from selected IMFs was used for the evaluation of pipelines utilizing the magnetic flux leakage (MFL) technique [14]. EEMD was also been used to simulate cardio-respiratory signals in order to measure cardiac stroke volume. EEMD improved them better than EMD by mode mixing removal [15].

Arrhythmia ECGs have different ECG patterns than the normal state. Different arrhythmia states, such as premature arrhythmias, superavent arrhythmias, ventricular arrhythmias and conduction arrhythmias, present various ECG waveforms. During the ECG measurement, various types of noises, such as muscle noise, baseline wander, and power-line interferences, are recorded in the ECG signals, interfering with the ECG-information identification. Numerous signal-processing methods have been used on the studies of ECG noise reduction, especially on arrhythmia ECGs. Adaptive regression and the corresponding Kalman recursions were used to remove ventricular fibrillation (VF) electrocardiogram (ECG) signal noise [16]. Multichannel Wiener filter and a matching pursuit-like approach were applied to remove cardiopulmonary resuscitation artifacts from human ECGs [17]. The adaptive LMS filter used to remove cardiopulmonary resuscitation (CPR) artifacts from ECGs has achieved high sensitivity and specificity of around $95 \%$ and $85 \%$, respectively [18]. Another adaptive filter based filter to suppress random noise in electrocardiographic (ECG) signals, unbiased and normalized adaptive noise reduction, can effectively eliminate random noise in ambulatory ECG recordings, leading to a higher SNR improvement than possible with a traditional LMS filter [19]. The time-frequency plane was also used to separate signal and noise components with an entire ensemble of repetitive ECG records, based on a Wiener filter. High noise reduction and low signal distortion was achieved after ensemble averaging problem involving repetitive deterministic signals mixed with uncorrelated noise [20].

The goal of this study is to investigate EEMD based filtering performance and the corresponding phase delay of filtered signals in arrhythmia ECGs. Low pass, high pass and band pass filters were 
designed to meet various noises conditions: muscle contraction, $50 \mathrm{~Hz}$ power line and baseline wonder. Traditional Butterworth filter and Wiener filter was also used to compare the filtering performance. Phase distortion of the filtered ECG was also investigated.

\section{EMD and EEMD algorithm}

\subsection{EMD}

The EMD algorithm used in this study comprises the following steps [1]:

(1) Identify all the extrema (maxima and minima) of the signal, $x(s)$.

(2) Generate the upper and lower envelope by the cubic spline interpolation of the extrema point developed in step (1).

(3) Calculate the mean function of the upper and lower envelope, $m(t)$.

(4) Calculate the difference signal $d(t)=x(t)-m(t)$.

(5) If $d(t)$ becomes a zero-mean process, then the iteration stop and $d(t)$ is an IMF1, named $c_{1}(t)$; otherwise, go to step (1) and replace $x(t)$ with $d(t)$.

(6) Calculate the residue signal $r(t)=x(t)-c_{1}(t)$.

(7) Repeat the procedure from steps (1) to (6) to obtain IMF2, named $c_{2}(t)$. To obtain $c_{n}(t)$, continue steps (1)-(6) after $n$ iterations. The process is stopped when the final residual signal $r(t)$ is obtained as a monotonic function.

At the end of the procedure, we have a residue $r(t)$ and a collection of $n \mathrm{IMF}$, named from $c_{1}(t)$ to $c_{n}(t)$. Now, the original signal can be represented as:

$$
x(t)=\sum_{i=1}^{n} c_{i}(t)+r(t)
$$

Often, we can regard $r(t)$ as $c_{n+1}(t)$.

\subsection{EEMD}

According to $\mathrm{Wu}[9]$, the steps for the EEMD algorithm are as follows:

(1) Add a white noise series $n(t)$ to the targeted signal, named $x_{1}(t)$ in the following description, and $x_{2}(t)=x_{1}(t)+n(t)$.

(2) Decompose the data $x_{2}(t)$ by EMD algorithm, as described in Section 2.1.

(3) Repeat Steps (1) and (2) until the trial numbers, each time with different added white noise series of the same power at each time. The new IMF combination $C_{i j}(t)$ is achieved, where $i$ is the iteration number and $j$ is the IMF scale.

(4) Estimate the mean (ensemble) of the final IMF of the decompositions as the desired output:

$$
E E M D_{-} c_{j}(t)=\sum_{i=1}^{n i} c_{i j}(t)
$$

where $n i$ denotes the trial numbers. 


\section{Method}

A simulated arrhythmia ECG segment with designed noises was used to examine filter output. Noises contained EMG, $50 \mathrm{~Hz}$ power line and baseline wanders. Low pass filter, high pass filter and band pass filters were designed with Butterworth filter, Wiener filter, EMD and EEMD based filters. The filtering performance was compared. The overall flowchart is shown in Figure 1. The detailed description is given in the following sub-section.

Figure 1. Flowchart of this study.

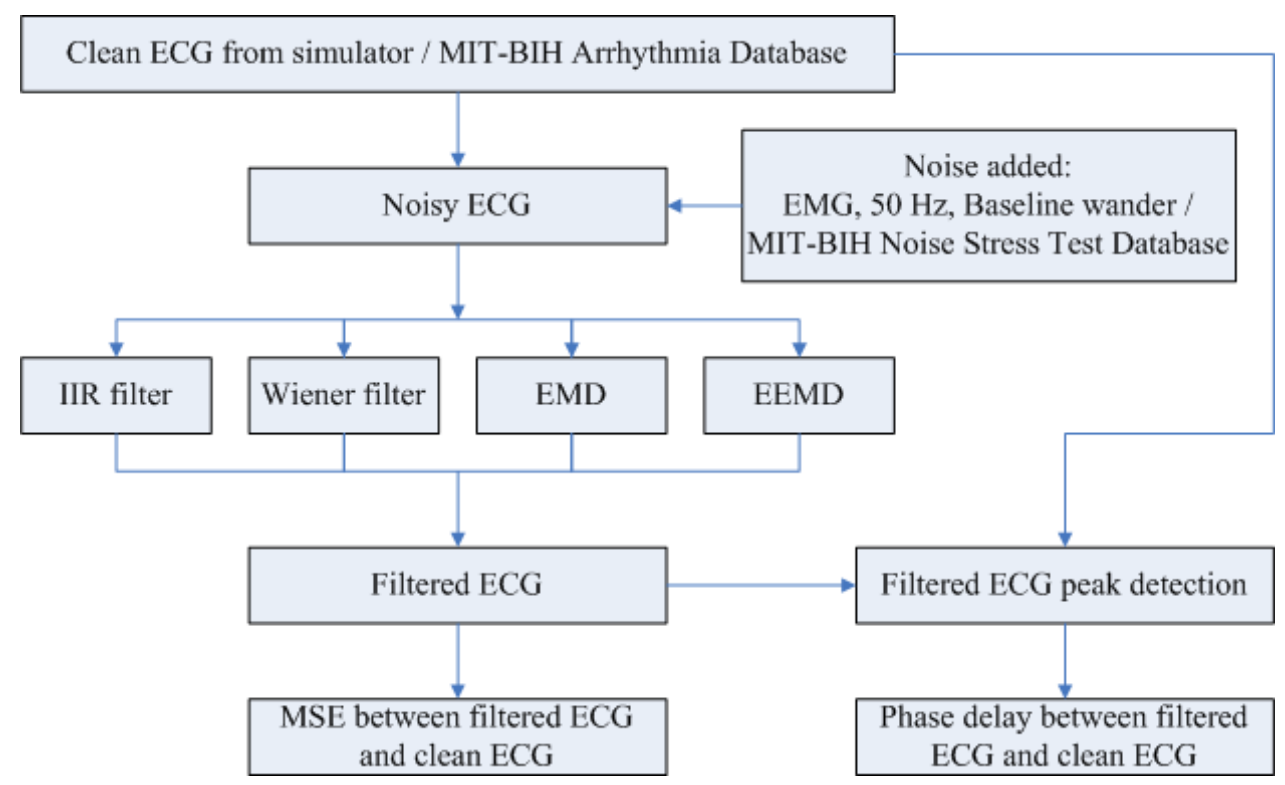

\subsection{Simulated Arrhythmia ECG and Noise Data}

A. Clean synthetic ECG signal:

Simulated normal and arrhythmia ECGs were derived from a ECG simulator (type number BC Biomedical PS-2210 Patient Simulator) with $60 \mathrm{~s}$ duration. The ECG simulator parameter was $80 \mathrm{BPM}$, temperature $37{ }^{\circ} \mathrm{C}$, Maximum peak to minimum peak voltage was $5 \mathrm{mV}$, breath Rate was set at 30. There are one normal ECG, and six arrhythmia ECGs, such as premature arrhythmias, superavent arrhythmias, ventricular arrhythmias and conduction arrhythmias. The ECG segment is shown in Figure 2. The corresponding ECG label and disease type was also described in the caption of Figure 2.

B. Real ECG database

Real ECG data was derived from an arrhythmia ECG database. Number 101, 102 and 103 and 104 were used. A band-pass filter ranged $1-35 \mathrm{~Hz}$ was used as preprocessing filter. The cleaned ECG was then used a real ECG template. The signal was 30 min durations. [21]. 
Figure 2. Illustration of normal and arrhythmia ECG signals used in this study. Signal durations are $30 \mathrm{~s}$. From top to bottom: (a) normal ECG, (b) premature arrhythmia with PVC1, denoted as P1, (c) premature arrhythmia with multifocal PVC, denoted as P2, (d) superavent arrhythmia with atrial tach, denoted as S1, (e) superavent arrhythmia with sinus arrhythmia, denoted as S2, (f) ventricular arrhythmia with 24 PVCs per min, denoted as $\mathrm{V} 1,(\mathrm{~g})$ conduction arrhythmia with Lf bundle branch block, denoted as C1.

(a)

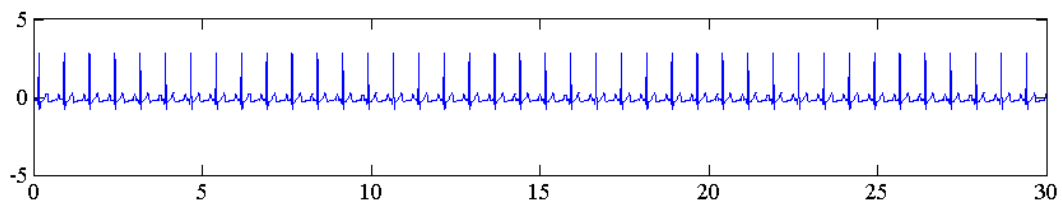

(b)

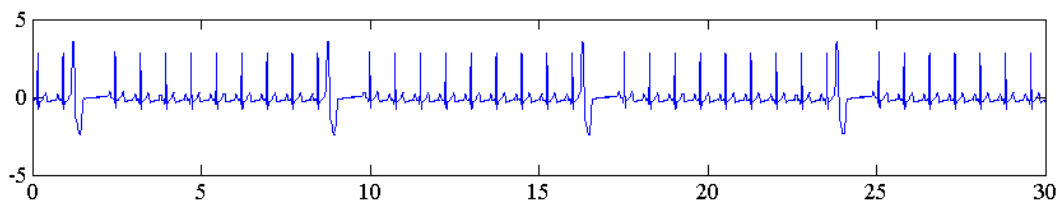

(c)

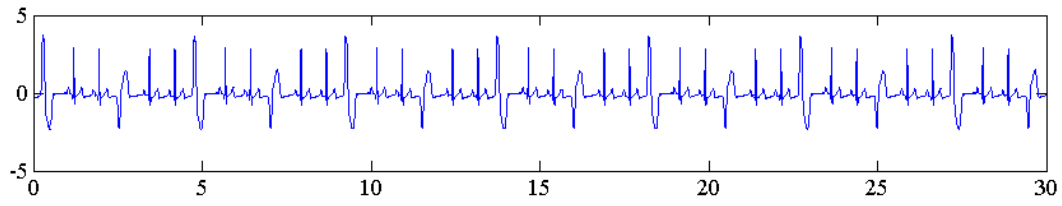

(d)

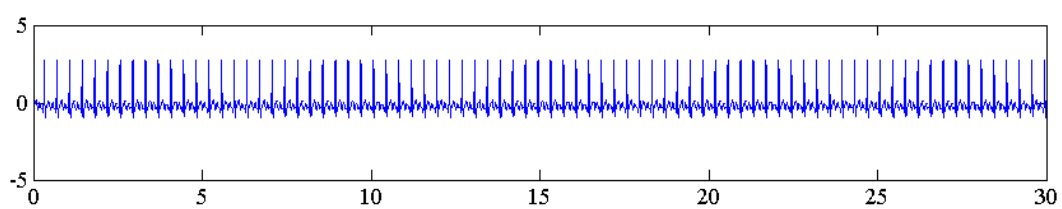

(e)

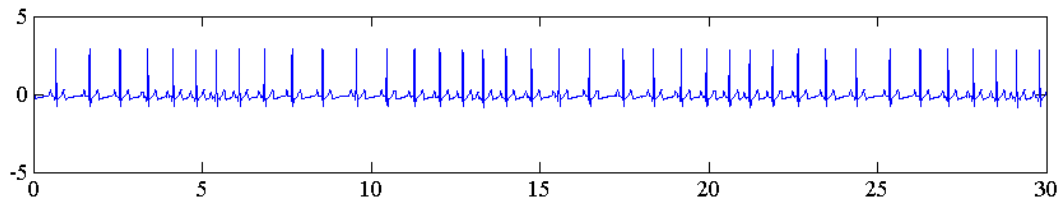

(f)

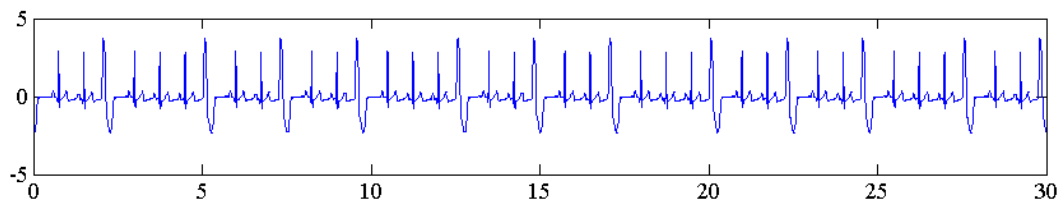

(g)

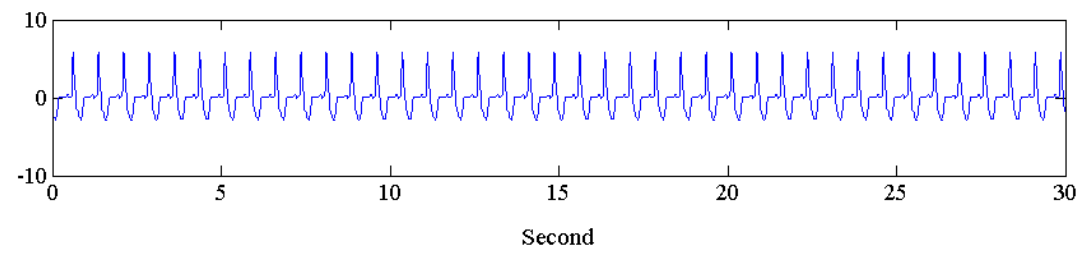

C. Synthetic noises:

High frequency ECG noise types, such as muscle contraction and $50 \mathrm{~Hz}$ power line interference, and low frequency ECG, baseline wander were investigated in the following session. All noises were also reduced to three noise levels, $25 \%, 50 \%$ and $100 \%$, with respect to the maximum noise level. The maximum noise level was predetermined as an amplitude ratio with respect to normal ECG, Vpp, 
which is amplitude of maximum peak to minimum peak. The noise simulation algorithm was similar to the suggestion in [22]:

1. EMG noise: EMG noise was model by a random number with normal distribution, originally manipulated with the Matlab code randn.m. The maximum EMG noise level was the scaling of random sequence and the multiplication to $\mathrm{Vpp}$ with reduced ratio of 1/8. EMG noise sequence was denoted as N1(t).

2. Power line noise: Power line interference was modeled by $50 \mathrm{~Hz}$ sinusoidal function with multiplication on amplitude derived with Matlab code rand.m. The maximum $50 \mathrm{~Hz}$ noise level was the scaling of random sequence and the multiplication to $\mathrm{Vpp}$ with reduced ratio of $1 / 4.50 \mathrm{~Hz}$ noise sequence was denoted as $\mathrm{N} 2(\mathrm{t})$.

3. Baseline wander: Baseline wander was model by a Baseline wander a $0.333 \mathrm{~Hz}$ sinusoidal function. The maximum noise level was the same amplitude scale with Vpp. Baseline wander was denoted as $\mathrm{N} 3(\mathrm{t})$.

4. Composite noise: Composite noise was the combination of the above three noise with the following relation :

$$
\mathrm{N} 4(\mathrm{t})=0.5^{*}[\mathrm{~N} 1(\mathrm{t})+\mathrm{N} 2(\mathrm{t})]+\mathrm{N} 3(\mathrm{t})
$$

Illustration of the four noises with three levels on a normal ECG is shown in Figure 3.

Figure 3. Illustration of EMG noise, $50 \mathrm{~Hz}$ noise, baseline wander and composite of three noises on normal ECG. Noise levels of 25\%, 50\% and 100\% are added, respectively. Signal durations are $3 \mathrm{~s}$.
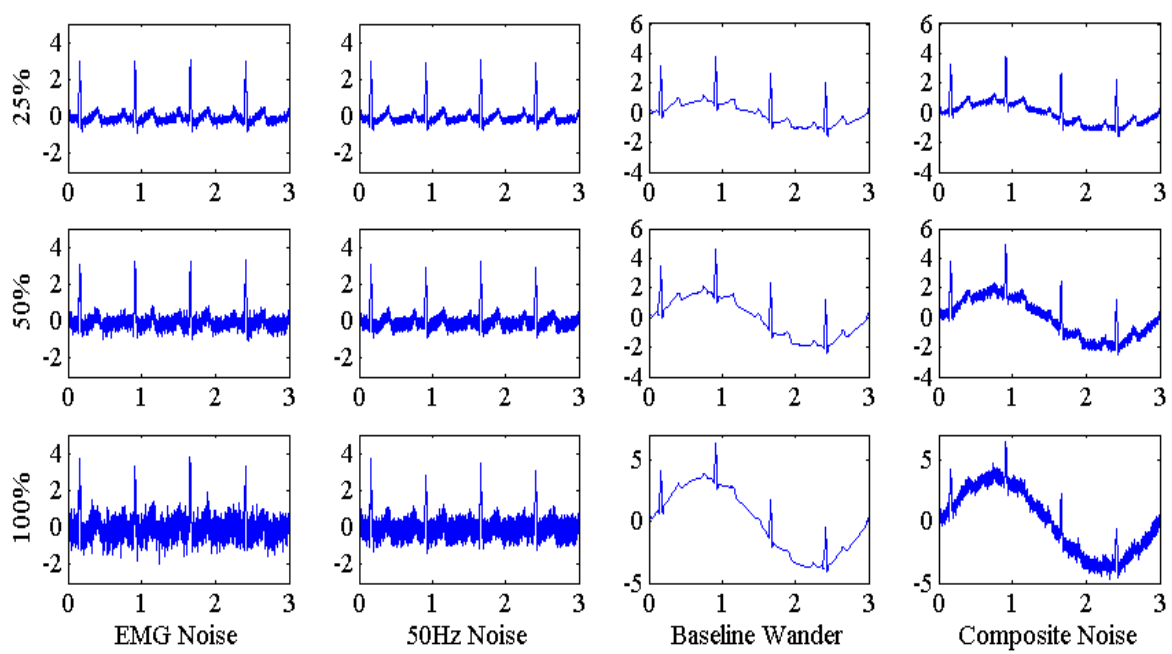

D. Real noise database

Real noises are extracted from the noise stress test database in MIT-BIH [23]. There are three noise patterns: baseline wander (in record "bw"), muscle (EMG) artifact (in record "ma"), and electrode motion artifact (in record "em"). Both one minute and total 30 min duration noises were selected, respectively. The short one minute noise was used for synthetic ECG and the 30 min duration noise was for real ECG signal derived from the arrhythmia ECG database. 


\subsection{EMD/EEMD Based Filtering Algorithm}

ECG was filtered (reconstructed) with partial reconstruction IMF by EMD, EEMD respectively with following equation:

$$
\begin{gathered}
R E C G \_e m d_{k q}(t)=\sum_{i=k}^{q} c_{i}(t), \\
R E C G \_e e m d_{k q}(t)=\sum_{i=k}^{q} E E M D{ }_{-} c_{i}(t),
\end{gathered}
$$

When $k=1, \mathrm{q}=\mathrm{n}, \mathrm{RECG} \_\mathrm{emd}_{1 \mathrm{q}}$ becomes equivalent to the original noised ECG. A low pass filter was derived from deletion of lower IMF scale, than means $\mathrm{k}>1$; A high pass filter was derived from deletion of high IMF scale, than means $\mathrm{q}<\mathrm{n}$; and a band pass filter was consequently with middle part of IMF scales, that means both conditions $\mathrm{k}>1$ and $\mathrm{q}<\mathrm{n}$ must be satisfied. The optimal choice of $(\mathrm{k}, \mathrm{q})$ pairs for each filter was determined with minimum MSE by sequential search approach. The EEMD parameters was $10 \mathrm{~dB}$ added white noise and 200 times trial number, according to previous study [24].

\subsection{Wiener Filter}

The formula of the Wiener filter is given as [25]:

$$
w=R_{X_{1} X_{1}}^{-1} R_{X_{1} X}
$$

where $w$ is the Wiener filter coefficients, and the cross correlation of $x_{1}(t)$ and $x(t), R_{X_{1} X}$, autocorrelation of $x_{1}(t), R_{X_{1} X_{1}}$ were estimated. The $x_{1}(t)$ and $x(t)$, represent the input signal and desired signal corresponding to $x_{1}(t)$ and $x(t)$ introduced in the earlier section, respectively. Wiener filter theory is based on the minimization of difference between the filtered output and desired output. Filter coefficient was estimated by the least mean squares method on the square of the difference between the desired and the actual signal after filtering. In this study, the Wiener filter was derived from Matlab function firwiener.m, with filter order 300.

\subsection{Traditional IIR Filter}

A Butterworth filter was used with three filter speculation. The low pass filter was a 10th order Butterworth filter with a $35 \mathrm{~Hz}$ cutoff frequency, and the high pass filter was a 3rd order Butterworth filter with a $1 \mathrm{~Hz}$ cutoff frequency. The band pass filter was the cascade computation result of the low pass filter and high pass filter.

\subsection{Filtering Performance Index}

Three are two indexes used to indicate the filter performance on EEMD and the other filter, one is mean square error (MSE) and the other is phase delay. MSE was to measure the difference between the original "clean" ECG and the reconstructed ECG. MSE is mainly from the residual noise and also ECG distortion after filtering process. MSE can be defined as follows: 


$$
M S E=\frac{\sum_{t=0}^{L-1}(x(t)-\hat{x}(t))^{2}}{L}
$$

where the nominator part is the square error, and $\hat{x}(t)$ is the reconstructed ECG, such as $R E C G_{-} e m d_{k q}$ or $R E C G_{-} e e m d_{k q}$ in Equations (4) and (5). The phase delay of $\hat{x}(t)$ was also calibrated before MSE calculation. $L$ is the length of the signal. The lower the MSE value, the higher filtering performance was evaluated for filters. Another quantitative feature, $\mathrm{MSE}_{\mathrm{QRS}}$ is also defined as the MSE within the windowed QRS complex:

$$
M S E_{Q R S}=\frac{\sum_{k=1}^{L} \sum_{t=0}^{W-1}(y(t)-\hat{y}(t))^{2}}{L^{*} W}
$$

where $\mathrm{L}$ is the number of QRS complex, and $\mathrm{W}$ is the window duration of each QRS complex. $\mathrm{MSE}_{\mathrm{QRS}}$ is used to measure the recovery performance of QRS complex with various filter method.

\section{Results:}

\subsection{EMD and EEMD Decomposition:}

The typical EMD and EEMD decomposition and extracted IMF are illustrated in Figure 4. The low level IMF contained high frequency components; while the high level IMF contained low frequency components. IMF distribution is very similar to a filter bank. Unlike a traditional filter bank, and similar sub-band decomposition algorithms, such as wavelets, IMF is not band restricted. Adaptive decomposition based on the signal pattern complexity is the main feature of IMF. Not specific IMF level would contain pre-determined frequency range components, that means an adaptive frequency range filtering process.

Figure 4. Illustration of IMF distribution of a ventricular arrhythmia ECG (V1) with 100\% composite noise by: (a) EMD, and (b) EEMD. From top to bottom is low level IMF to high level IMF. Signal durations are $30 \mathrm{~s}$.

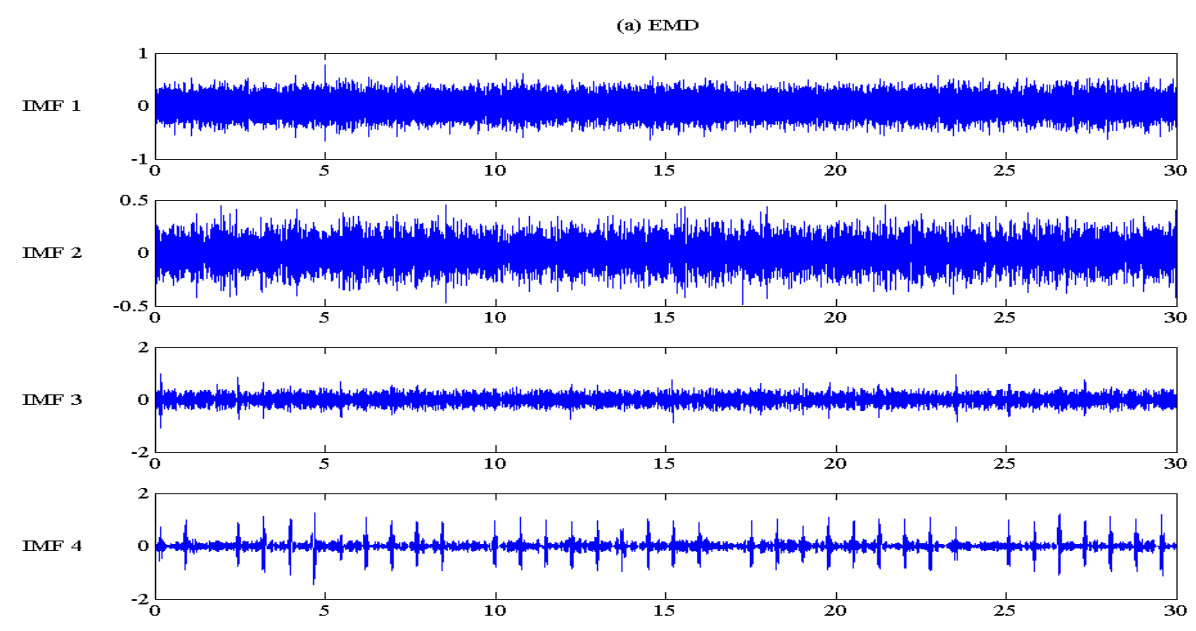


Figure 4. Cont.

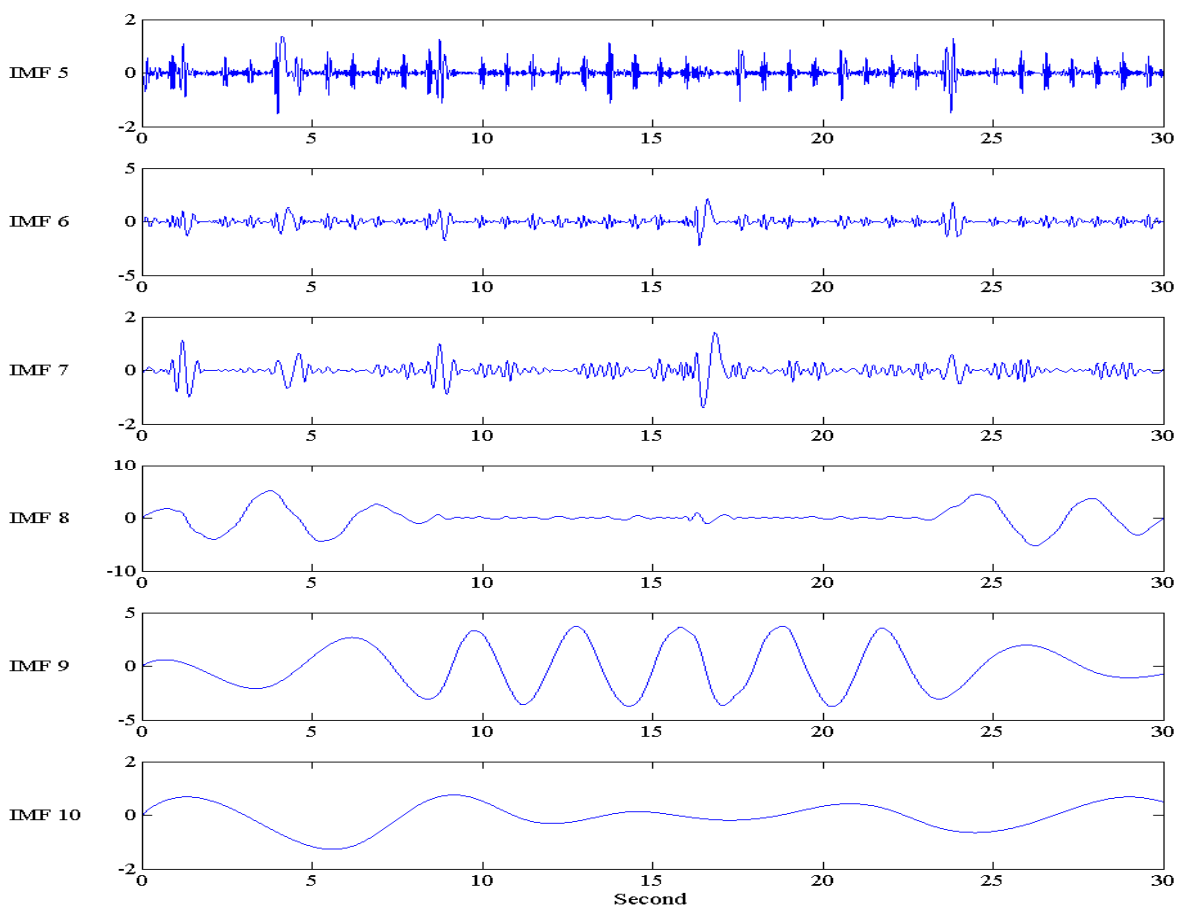

(b) EEMD
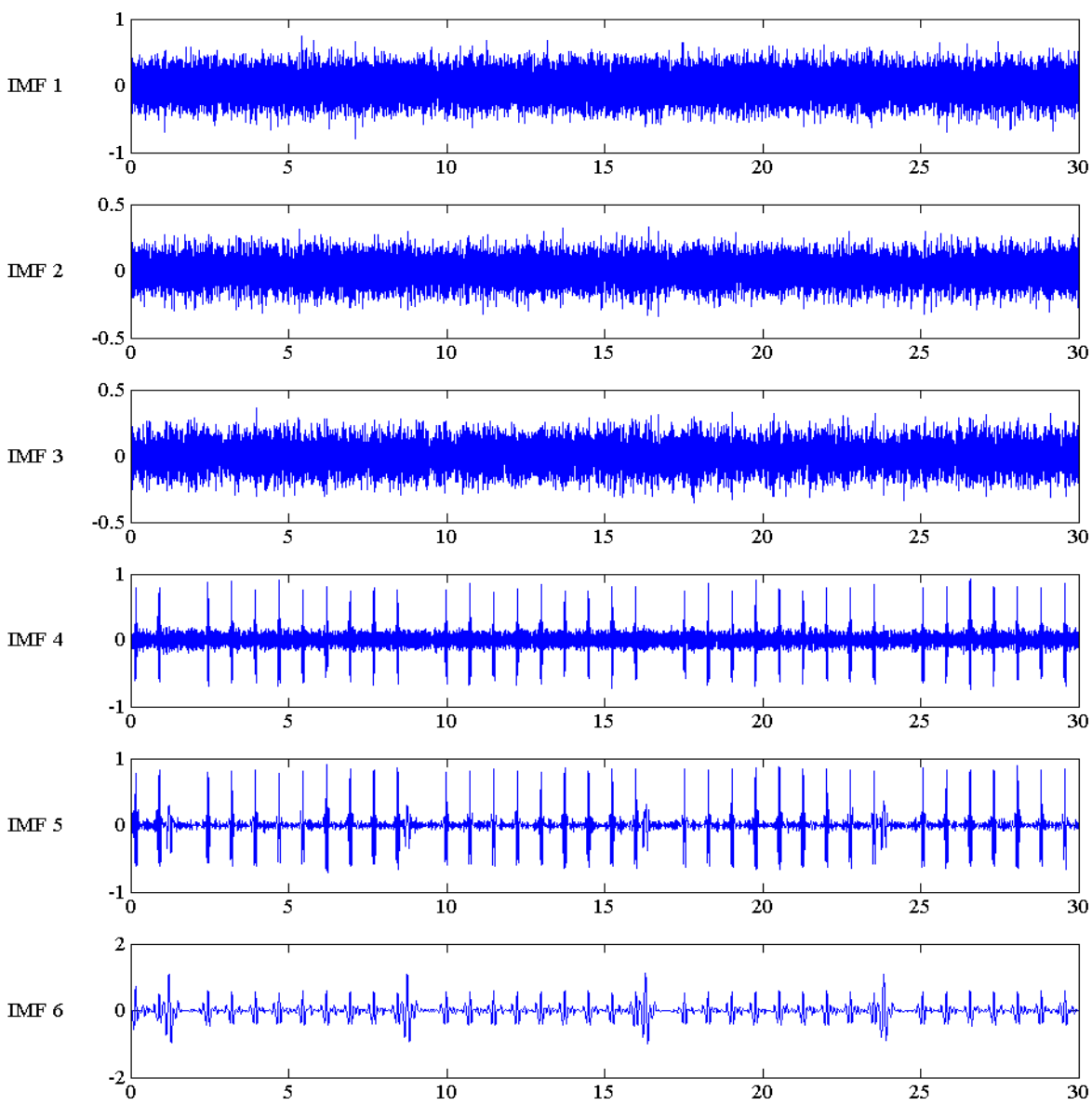

IMF 720 find 
Figure 4. Cont.
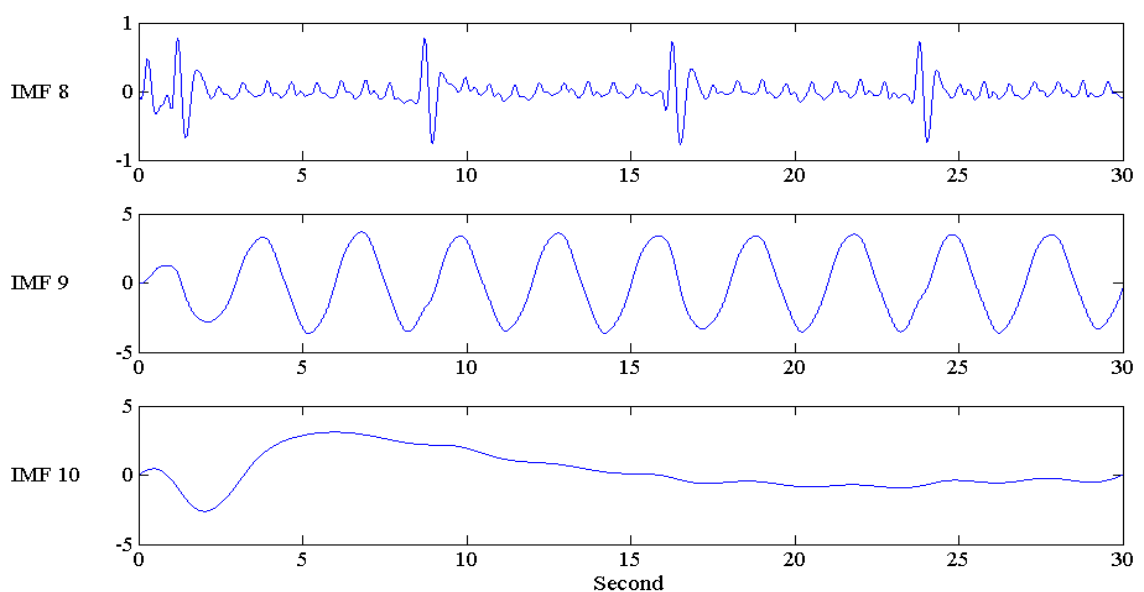

The difference between EMD and EEMD is the mode mixing reduction of EEMD. Comparing the IMF component of the same level, EEMD has more concentrated and band limited components. High frequency noises are more localized in the low IMF level. That can be seen from Figure 5, the corresponding IMF spectrum distribution of EMD and EEMD. The $50 \mathrm{~Hz}$ spike is in the 1st-4th level in EMD and EEMD; while the $0.33 \mathrm{~Hz}$ baseline wander is in the 8th and 9th level in EMD and only in 9th level in EEMD. ECG components are located between the 4th to 7th level in EMD and 4th to 8th level in EEMD.

Figure 5. Corresponding IMF spectrum distribution of (a) EMD, and (b) EEMD of Figure 4. The $\mathrm{x}$-axis unit is $\mathrm{Hz}, \mathrm{y}$-axis is power. There is less spectrum overlapping of EEMD than EMD due to reduction of mode mixing in EMD.

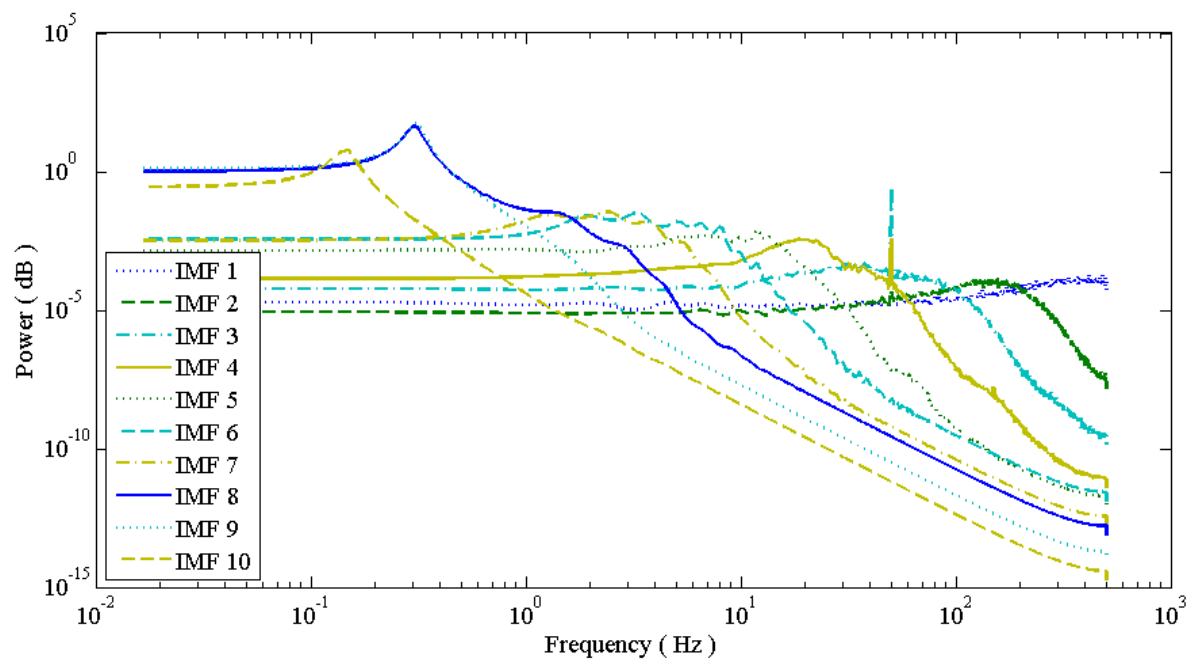


Figure 5. Cont.

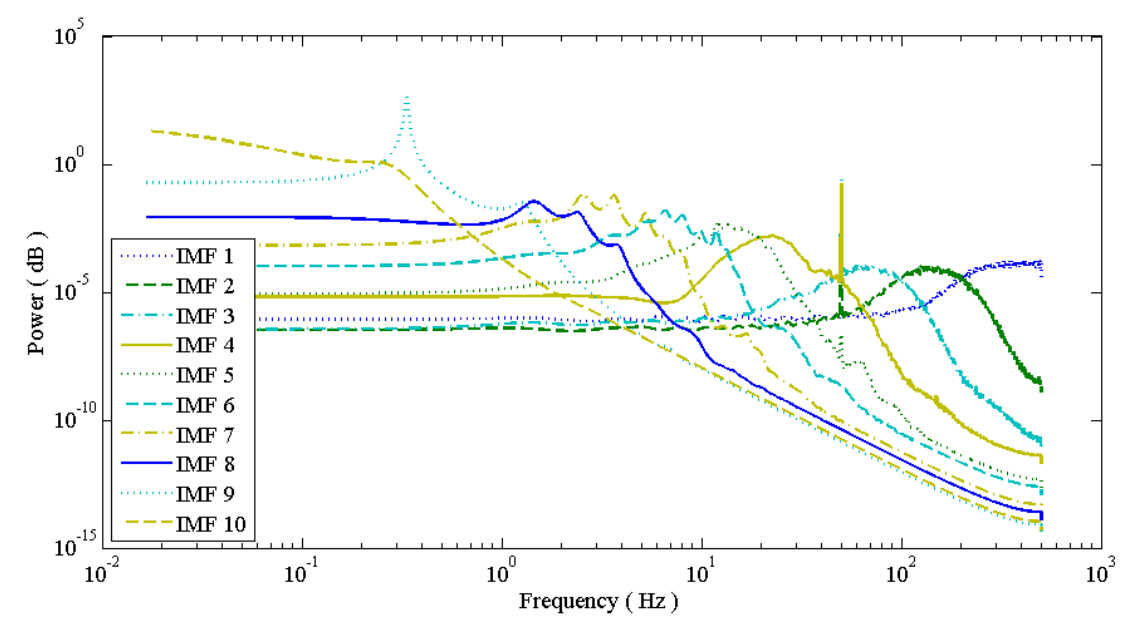

\subsection{MSE Performance:}

Noise reduction performance was evaluated by MSE. MSE performance of low pass filter and high pass filter spec is represented in Figure 6(a) and Figure 6(b). As shown in Figure 6(a), as IMF level increased, MSE value would be decreased due to the remove of high frequency noise components; while $\mathrm{k}$ increased and signal components were also be deleted, MSE increased due to signal distortion. Therefore the optimal IMF level was chosen on the concave with minimum MSE value, and an EMD/EEMD based low pass filter was determined. For the same reason, a high pass filter was also determined with another concave with the deletion from high IM level.

Figure 6. MSE distribution of ventricular arrhythmia ECG (V1) with (a) 25\% EMG noise , (b) $100 \%$ baseline wander for EMD (dot line with triangle mark), EEMD (dot line with square mark),on different IMF levels. Corresponding MSE of Wiener filter (dash line) and IIR filter (solid line) with low pass filter spec are also shown in (a) and in (b) with high pass filter spec. The minimum MSE of EMG noise is at $\mathrm{k}=3$ for EMD and $\mathrm{k}=4$ for EEMD, and minimum MSE of baseline wander is at $q=8$ (EEMD) and $q=4$ (EMD), respectively.

(a) $25 \%$ EMG noise

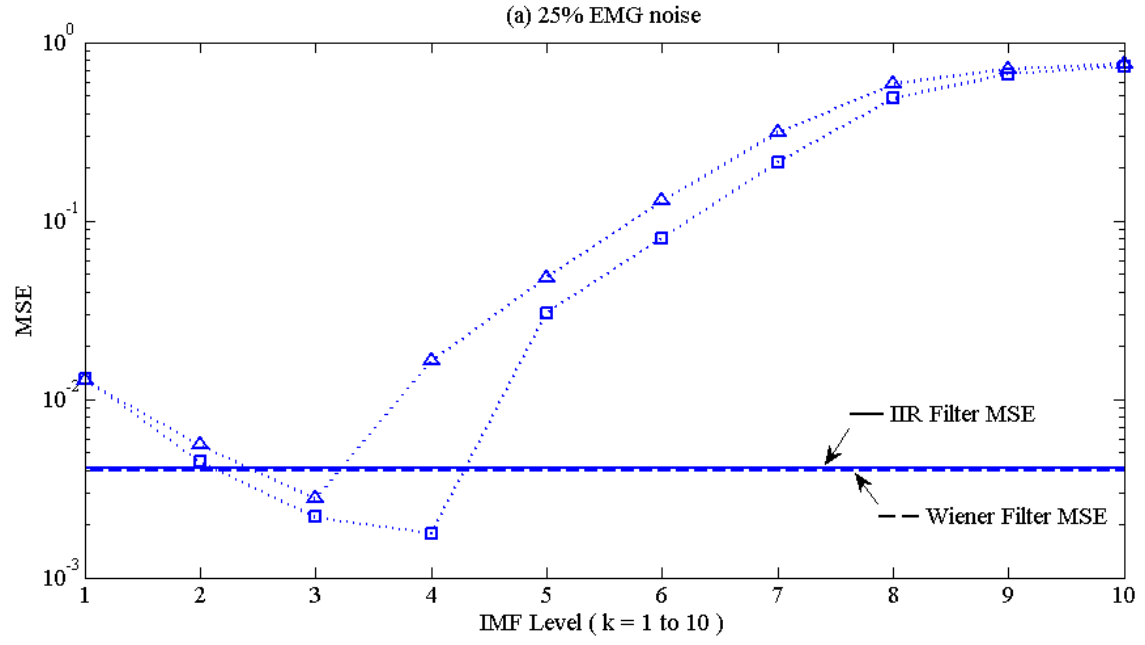


Figure 6. Cont.

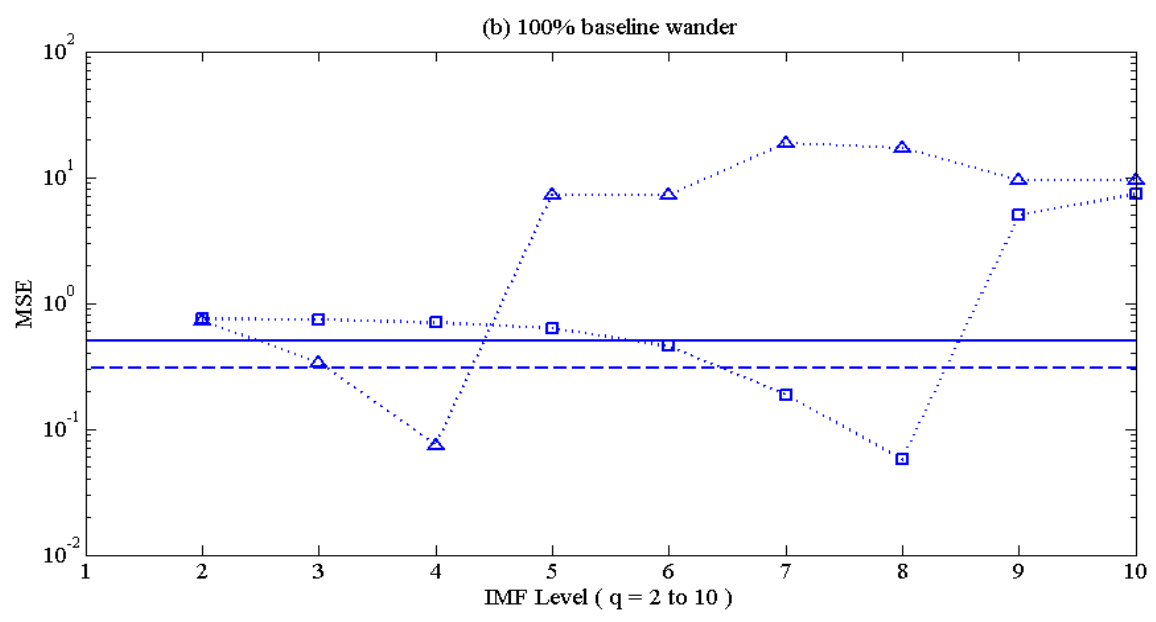

EMD and EEMD based band pass filter need the reduction of both high level and low level IMF, and optimally the middle part of IMF would be conserved, that corresponds to the clean ECG component. Sequential search of MSE with all possible $(k, q)$ combinations was evaluated. A contour map with $\mathrm{x}$-axis as $\mathrm{k}$ and $\mathrm{y}$-axis as $\mathrm{q}$ was sketched. A minimum MSE point to indicated the optimal $(\mathrm{k}, \mathrm{q})$ pair location is achieved for the optimal band pass filtering performance and is illustrated in Figure 7.

Figure 7. Contour map of MSE under various (k,q) pairs for Ventricular arrhythmia ECG (V1) with $100 \%$ composite noise by EEMD. The (k,q) location with lowest MSE was triangle mark. In this case, $\mathrm{k}=4$ and $\mathrm{q}=8$ was the optimal solution.

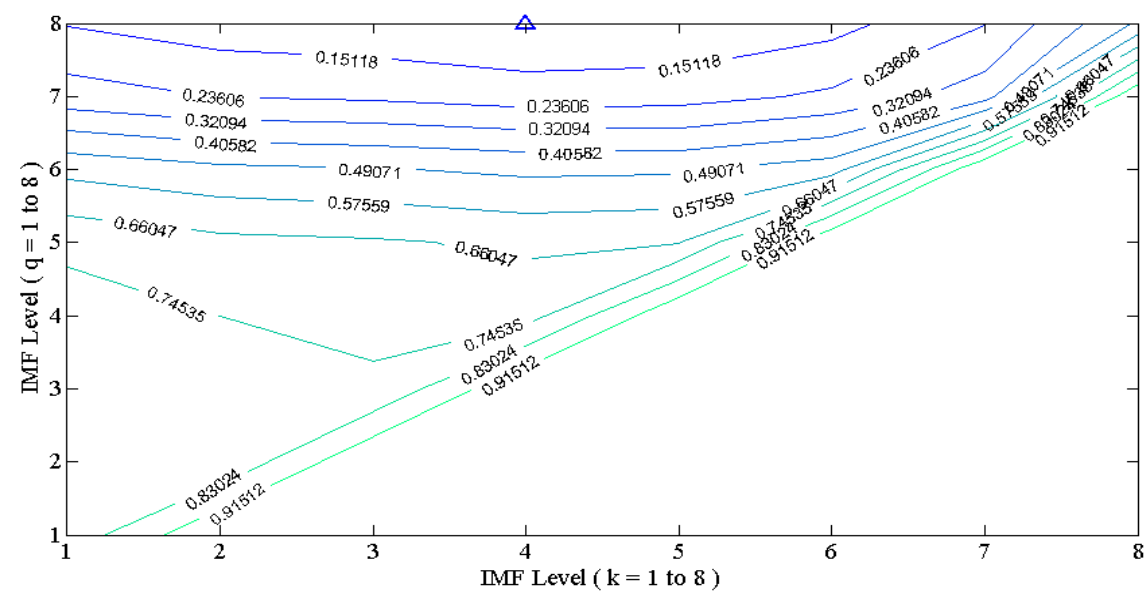

Figure 6 shows that the MSE ranking from high to low was IIR > Wiener $>$ EMD $>$ EEMD. EEMD always has lowest MSE under various noise contamination scenarios, which perform as low pass filter, high pass filter and also as band pass filter. This result, indicating that EEMD is also superior to other filters, not only for normal ECG, but also performs well for arrhythmia ECG, is also shown in Figure 8. That means EEMD has good noise reduction performance, under various ECG patterns. With the deletion of low IMF level, EEMD performed as a low pass filter; while with the deletion of high IMF 
level, EEMD performed as high pass filter. Sequential search of lowest MSE on (k,q) pairs also indicated the optimal band pass filter performance.

Figure 8. MSE percentage of all ECG contaminated with $100 \%$ composite noise. $\mathrm{X}$-axis is the seven ECG segments, the same sequence with that shown in Figure 2. Each ECG segment has average MSE of four filters, from left to right sites are IIR, Wiener, EMD and EEMD. EEMD always has lowest MSE percentage among the four filters, and it is always true for normal ECG, and also for arrhythmia ECG.

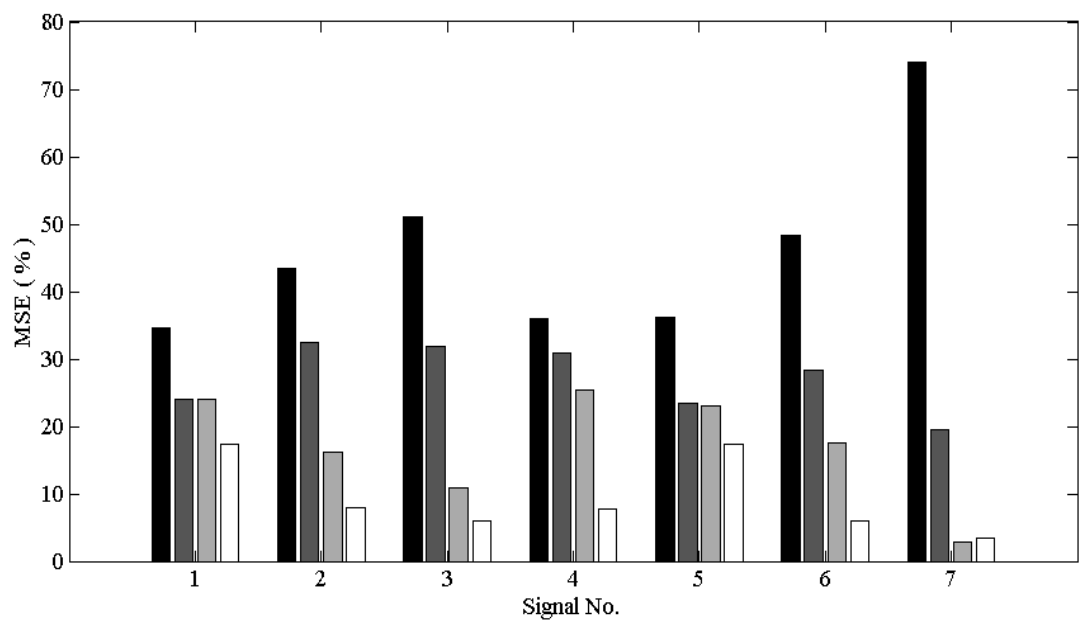

The detailed MSE values for synthetic ECG are listed in Table 1. From the results of Table 1, EEMD performed better with light noise percentage, and also better on baseline wander than on high frequency noise, both on $50 \mathrm{~Hz}$ interference and EMG noise.

Table 1. MSE result of Ventricular arrhythmia ECG of four filter methods with four noises. Minimum MSE of the same noise are mark bold.

\begin{tabular}{|c|c|c|c|c|c|}
\hline Noise type & $\begin{array}{c}\text { Noise } \\
\text { percentage }\end{array}$ & IIR & Wiener & $\begin{array}{c}\text { EMD } \\
\text { (IMF level) }\end{array}$ & $\begin{array}{c}\text { EEMD } \\
\text { (IMF level) }\end{array}$ \\
\hline EMG (* E-3) & $25 \%$ & 4.1 & 4.0 & $2.8(\mathrm{k}=3)$ & $\mathbf{1 . 8}(\mathrm{k}=4)$ \\
& $50 \%$ & 6.6 & 12.3 & $11.1(\mathrm{k}=4)$ & $\mathbf{4 . 6}(\mathrm{k}=4)$ \\
& $100 \%$ & 18.1 & 34.4 & $24.6(\mathrm{k}=4)$ & $\mathbf{1 8 . 1}(\mathrm{k}=4)$ \\
\hline $50 \mathrm{~Hz}(* \mathrm{E}-3)$ & $25 \%$ & 3.3 & $\mathbf{1 . 0}$ & $7.2(\mathrm{k}=2)$ & $2.0(\mathrm{k}=4)$ \\
& $50 \%$ & 3.8 & 3.0 & $11.9(\mathrm{k}=4)$ & $\mathbf{3 . 0}(\mathrm{k}=4)$ \\
& $100 \%$ & 5.7 & 9.4 & $10.7(\mathrm{k}=4)$ & $\mathbf{5 . 1}(\mathrm{k}=4)$ \\
\hline Baseline $(* \mathrm{E}-2)$ & $25 \%$ & 49.5 & 10.1 & $3.0(\mathrm{q}=5)$ & $\mathbf{2 . 3}(\mathrm{q}=9)$ \\
& $50 \%$ & 49.7 & 18.4 & $8.5(\mathrm{q}=5)$ & $\mathbf{4 . 8}(\mathrm{q}=8)$ \\
& $100 \%$ & 50.5 & 30.4 & $7.4(\mathrm{q}=4)$ & $\mathbf{5 . 7}(\mathrm{q}=8)$ \\
\hline Composite $(* \mathrm{E}-2)$ & $25 \%$ & 52.6 & 10.3 & $8.5(\mathrm{k}=3, \mathrm{q}=8)$ & $\mathbf{2 . 3}(\mathrm{k}=4, \mathrm{q}=9)$ \\
& $50 \%$ & 52.8 & 18.8 & $5.5(\mathrm{k}=3, \mathrm{q}=8)$ & $\mathbf{5 . 0}(\mathrm{k}=4, \mathrm{q}=8)$ \\
& $100 \%$ & 54.2 & 31.7 & $19.5(\mathrm{k}=4, \mathrm{q}=7)$ & $\mathbf{6 . 6}(\mathrm{k}=4, \mathrm{q}=8)$ \\
\hline "em"(* E-2) & $100 \%$ & 49.1 & 19.6 & $19.3(\mathrm{k}=1, \mathrm{q}=5)$ & $\mathbf{1 6 . 3}(\mathrm{k}=4, \mathrm{q}=7)$ \\
"ma" (* E-2) & $100 \%$ & 36.1 & 10.7 & $13.3(\mathrm{k}=3, \mathrm{q}=7)$ & $\mathbf{8 . 5}(\mathrm{k}=5, \mathrm{q}=9)$ \\
"bw" (* E-2) & $100 \%$ & 23.1 & 6.9 & $2.7(\mathrm{k}=1, \mathrm{q}=7)$ & $\mathbf{1 . 5}(\mathrm{k}=4, \mathrm{q}=9)$ \\
\hline
\end{tabular}


For real noises, baseline wander, muscle contraction and motion artifact, EEMD still had lowest MSE performance than other filters with synthetic V1 signal. The typical filtered ECG of the four filters used in this study is sketched in Figure 9. The IIR filtered ECG has some waveform distortion, especially on the S peak, and there is a pseudo positive peak on PVC pattern; while the output of EMD and EEMD remaining similar to the original ECG signal pattern. That is the advantage of EMD and EEMD with the near zero phase delay character.

Figure 9. From top to bottom: (a) ventricular arrhythmia ECG (V1) with 100\% composite noise and corresponding filter output by (b) IIR, (c) Wiener, (d) EMD $(\mathrm{k}=4, \mathrm{q}=7)$ and $(\mathrm{e}) \operatorname{EEMD}(\mathrm{k}=4, \mathrm{q}=8)$.

(a)

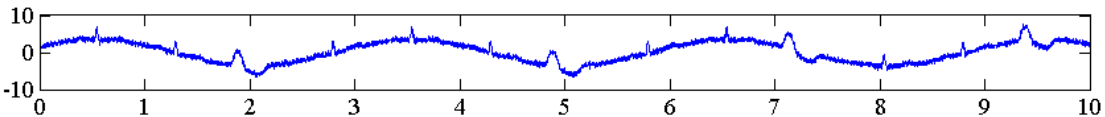

(b)

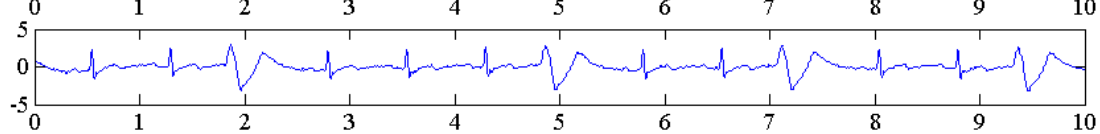

(c)

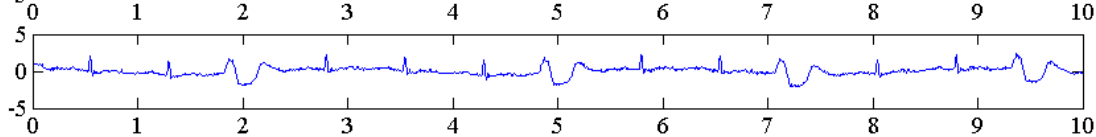

(d)

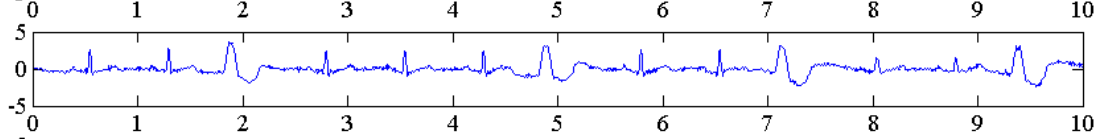

(e)

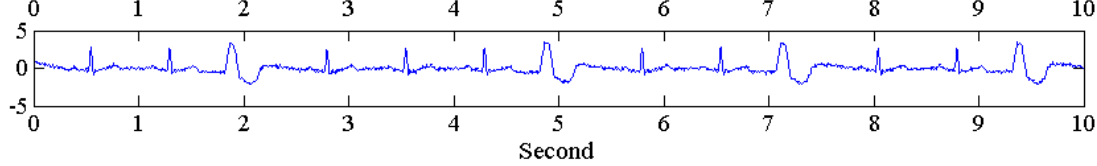

The filter output for V1 with real noise corruption is shown in Figure 10. It is obvious that EEMD has better filtering performance under muscle contraction contamination.

Figure 10. From top to bottom are (a) ventricular arrhythmia ECG (V1), (b) with muscle artifact 'ma' noise and corresponding filter output by (c) IIR, (d) Wiener, (e) EMD and (f) EEMD.

(a)

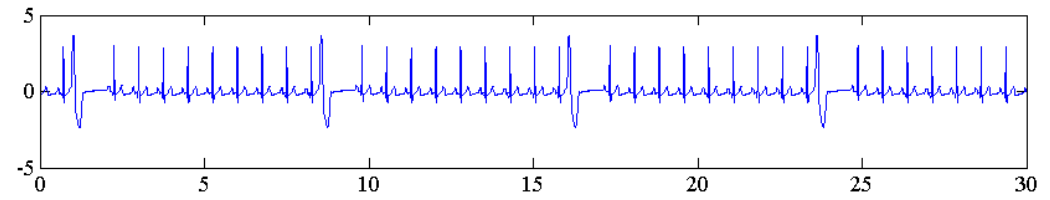

(b)

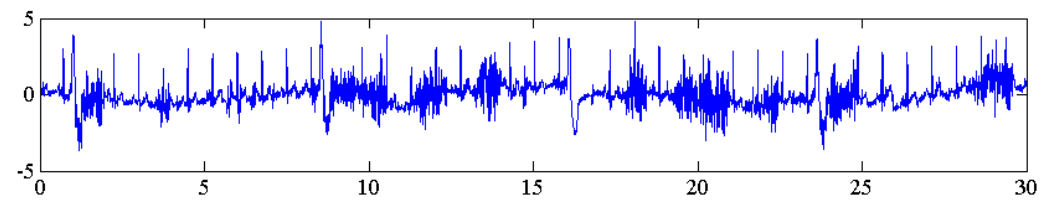

(c)

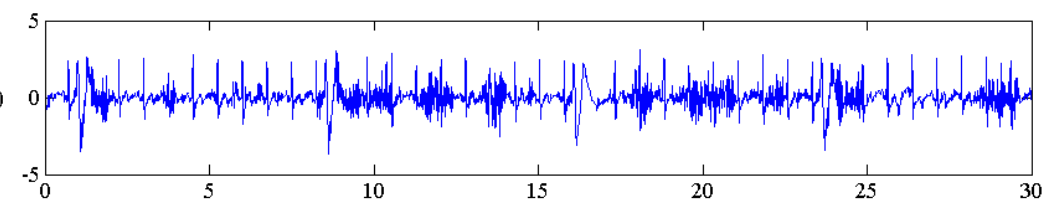


Figure 10. Cont.
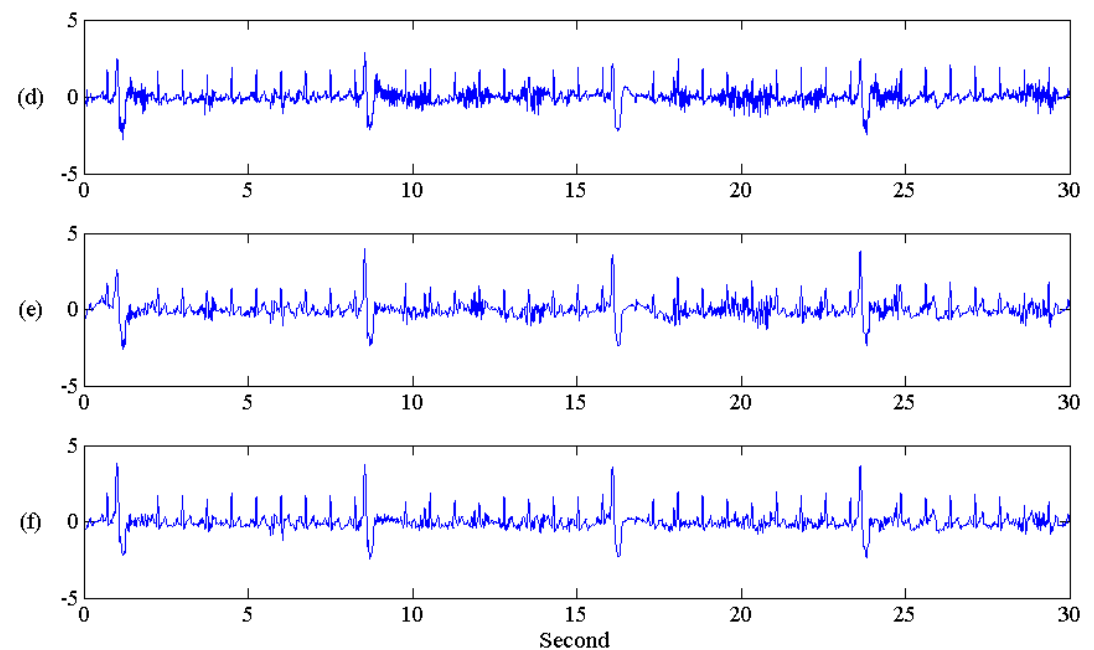

The QRS complex recovery for synthetic ECG is organized in Table 2. It is obvious from the data in this Table that MSE is higher with higher noise percentage. For baseline noise and composite noise, EEMD has lowest MSE than the other approach. There is no significant difference between the four filter methods on $\mathrm{MSE}_{\mathrm{QRS}}$ result for real noise contamination. The MSE performance of real noises on the real ECG database is shown in Table 3. Like Table 1, EEMD still has the lowest MSE than the other filters.

Table 2. $M S E_{\mathrm{QRS}}$ result of ventricular arrhythmia ECG of four filter methods with synthetic $100 \%$ composite noise and real noises. Minimum MSE of the same noise are mark bold.

\begin{tabular}{|c|c|c|c|c|c|}
\hline Noise type & $\begin{array}{c}\text { Noise } \\
\text { percentage }\end{array}$ & IIR & Wiener & EMD & EEMD \\
\hline \multirow{2}{*}{$\begin{array}{c}\text { EMG } \\
\text { * E-3) }\end{array}$} & $25 \%$ & $\mathbf{9 . 5}$ & 19.1 & 19.7 & 18.6 \\
\cline { 2 - 6 } & $50 \%$ & $\mathbf{1 2 . 0}$ & 65.1 & 75.8 & 67.7 \\
\cline { 2 - 6 } & $100 \%$ & $\mathbf{4 4 . 6}$ & 140.5 & 172.3 & 164.4 \\
\hline \multirow{2}{*}{$50 \mathrm{~Hz}$} & $25 \%$ & 8.6 & $\mathbf{2 . 1}$ & 8.4 & 3.4 \\
\cline { 2 - 6 }$(*$ E-3) & $50 \%$ & $\mathbf{9 . 1}$ & 26.2 & 41.4 & 27.8 \\
\cline { 2 - 6 } & $100 \%$ & 12.2 & $\mathbf{1 1 . 9}$ & 19.2 & 12.0 \\
\hline \multirow{2}{*}{$\begin{array}{c}\text { Baseline } \\
(* \text { E-2) }\end{array}$} & $25 \%$ & 53.4 & 9.3 & 2.4 & $\mathbf{1 . 6}$ \\
\cline { 2 - 6 } & $50 \%$ & 53.7 & 19.4 & 7.0 & $\mathbf{4 . 5}$ \\
\hline \multirow{2}{*}{$\begin{array}{c}\text { Composite } \\
(* \text { E-2) }\end{array}$} & $25 \%$ & 57.7 & 9.5 & 5.7 & $\mathbf{1 . 6}$ \\
\cline { 2 - 6 } & $50 \%$ & 58.2 & 20.3 & 5.1 & $\mathbf{4 . 8}$ \\
\cline { 2 - 6 } & $100 \%$ & 58.9 & 37.7 & 18.6 & $\mathbf{7 . 1}$ \\
\hline 'em'(* E-2) & $100 \%$ & 63.6 & $\mathbf{3 3 . 4}$ & 38.7 & 34.5 \\
\hline 'ma' (* E-2) & $100 \%$ & 54.9 & $\mathbf{4 1 . 4}$ & 55.7 & 50.8 \\
\hline 'bw' (*E-2) & $100 \%$ & $\mathbf{2 2 . 9}$ & 34.7 & 42.9 & 41.7 \\
\hline
\end{tabular}


Table 3. MSE result of real ECG of four filter methods with three real noises. Minimum MSE of the same noise are mark bold.

\begin{tabular}{|c|c|c|c|c|c|}
\hline Signal & Noise & IIR & Wiener & EMD & EEMD \\
\hline \multirow{3}{*}{$\begin{array}{c}101 \\
(* \mathrm{E}-2)\end{array}$} & em & 14.7 & 6.7 & 8.6 & $\mathbf{6 . 5}$ \\
\cline { 2 - 6 } & ma & 6.0 & 2.4 & 3.6 & $\mathbf{2 . 2}$ \\
\cline { 2 - 6 } & bw & 4.1 & 1.6 & 1.0 & $\mathbf{0 . 7}$ \\
\hline \multirow{2}{*}{$\begin{array}{c}102 \\
*\end{array}$} & em -2$)$ & 18.2 & $\mathbf{7 . 5}$ & 10.9 & 7.9 \\
\cline { 2 - 6 } & ma & 9.5 & 2.3 & 3.2 & $\mathbf{1 . 9}$ \\
\cline { 2 - 6 } & bw & 7.6 & 1.9 & 1.3 & $\mathbf{0 . 8}$ \\
\hline 103 & em & 13.7 & 6.2 & 7.3 & $\mathbf{5 . 7}$ \\
\cline { 2 - 6 }$(*$ E-2) & ma & 5.0 & $\mathbf{2 . 4}$ & 3.7 & 2.8 \\
\cline { 2 - 6 } & bw & 3.1 & 1.3 & 1.0 & $\mathbf{0 . 6}$ \\
\hline
\end{tabular}

\section{Discussion:}

This article investigated the effect of EEMD filtering both on normal ECG and arrhythmia ECG. In additional to normal ECG, EEMD seem more useful on arrhythmia ECG filtering. Arrhythmia ECG with composite noise is the most common case during clinical ECG measurement. Not only is the lower MSE performance, but also on conservation of filtered ECG waveform performed by EEMD. In this study, signal P1, P2, V1, C1 displayed impressive filtering advantages with EEMD, especially on the PVC peak. There are some pseudo peaks produced by the IIR filter, especially on the S peak and a pseudo PVC positive peak. These pseudo peaks could lead to an improper medical diagnosis.

Mode mixing reduction between adjacent IMF levels is the main advantage of EEMD over traditional filters on arrhythmia ECG noise filtering. With the higher computation effort, it leads to better filtering performance. Due to the added noise used during EEMD, there is better filtering performance for EEMD on low noise power conditions.

ECG noise reduction procedure by EEMD on arrhythmia ECG with composite noise was proposed in this article. Something similar has been proposed based on EMD [10], but EEMD had better filtering performance than EMD by reducing mode mixing. The previous study was devoted to the high frequency noises, this study has tried to include the baseline wander noise and extend the signal to arrhythmia ECG. The criterion to achieve an optimal EEMD level selection rule is also proposed. For low pass filtering, iterative deletion of low level IMF until a minimum MSE is reached. The same method is used for high pass filtering, but with deletion of high level IMF to reserve the high frequency component in the low IMF level. It is a little time-consuming to obtain the optimal band pass filtering criteria on suitable IMF levels, but it can be replaced by visual inspection with relative IMF components. From Figure 4 it can be seen that level 4 to level 7 on the EMD contain R peak information, seen in level 4 to level 8 in EEMD. Therefore a smart guess of $(k, q)$ pairs with slight IMF level modification may be necessary to achieve the minimum MSE points with less computation. In the future, optimal selection criteria of IMF level in an interesting issue. Since each IMF is a filter-like output, it is reasonable to expect a predictable IMF level for ECG noise reduction. Unfortunately, the frequency range of each IMF level is not "predictable", unlike traditional filter banks; therefore there is 
no criterion now to predict an optimal IMF level for noise reduction. This will be a challenging topic to be investigated in future work.

\section{Conclusions}

This paper proposes a high performance and easy implemented ECG noise reduction procedure based on EEMD. Application of EEMD with adaptive IMF basis properties also has potential for other biomedical signals or other fields. For arrhythmia ECG with PVC it is more useful to use EEMD to remove composite noise than traditional filters. Although EEMD has a heavy computational load, it is still suitable for getting better noise reduction performance on arrhythmia ECG under off line analysis.

\section{Acknowledgements}

This work has been partly supported by the National Science Council of Taiwan (grant number NSC 98-2221-E-468-009-) and Asia University (grant number 98-ASIA-06-1).

\section{References}

1. Huang, N.E.; Shen, Z.; Long, S.R.; Wu, M.C.; Shih, H.H.; Zheng, Q.; Yen, N.C.; Tung, C.C.; Liu, H.H. The empirical mode decomposition and the Hilbert spectrum for nonlinear and non-stationary time series analysis. Proc. Roy. Soc. Lond. 1998, 454, 903-995.

2. Flandrin, P.; Rilling, G.; Goncalves, P. Empirical mode decomposition as a filter bank. IEEE Signal Process. Lett. 2004, 11, 112-114.

3. Li, D.; Liang, Z.; Voss, L.J.; Sleigh, J.W. Analysis of depth of anesthesia with Hilbert-Huang spectral entropy. Clin. Neurophysiol. 2008, 119, 2465-2475.

4. Salisbury, J.I.; Sun, Y. Rapid screening test for sleep apnea using a nonlinear and nonstationary signal processing technique. Med. Eng. Phys. 2007, 29, 336-343.

5. Balocchi, R.; Menicucci, D.; Santarcangelo, E.; Sebastiani, L.; Gemigani, A.; Ghelarducci, B.; Varanini, M. Deriving the respiratory sinus arrhythmia from the heartbeat time series using empirical mode decomposition. Chaos Solitons Fractals 2004, 20, 171-177.

6. Weng, B.; Blanco-Velasco, M.; Barner, K.E. ECG Denoising based on the Empirical Mode Decomposition. Conf. Proc. IEEE. Eng. Med. Biol. Soc. 2006, 1, 1-4.

7. Lu, Y.; Yan, J.Y.; Yam, Y. Model-based ECG Denoising Using Empirical Mode Decomposition. Conf. Proc. IEEE BIBM 2009, 2009, 191-196.

8. Pan, N.; I V. M.; Un, M.P.; Hang, P.S. Accurate Removal of Baseline Wander in ECG Using Empirical Mode Decomposition. Conf. Proc. NFSI ICFBI 2007, 2007, 177-180.

9. Li, N.Q.; Li, P. An Improved Algorithm Based on EMD-Wavelet for ECG Signal De-noising. In Proceedings of International Joint Conference on Computational Sciences and Optimization 2009, Sanya, Hainan, China; 24-26 April 2009, Volume 1.

10. Blanco-Velasco, M.; Weng, B.; Barner, K.E. ECG signal denoising and baseline wander correction based on the empirical mode decomposition. Comput. Biol. Med. 2008, 38, 1-13.

11. Nimunkar, A.J.; Tompkins, W.J. EMD-based 60-Hz noise filtering of the ECG. Conf. Proc. IEEE Eng. Med. Biol. Soc. 2007, 2007, 1904-1907. 
12. Wu, Z.; Huang, N.E. A study of the characteristics of white noise using the empirical mode decomposition method. Proc. Roy. Soc. London. A 2004, 460, 1597-1611.

13. Wu, Z.; Huang, N.E. Ensemble empirical mode decomposition: a noise-assisted data analysis method. Adv. Adapt. Data. Anal. 2009, 1, 1-41.

14. Chen, L.; Li, X.; Li, X.B.; Huang, Z.Y. Signal extraction using ensemble empirical mode decomposition and sparsity in pipeline magnetic flux leakage nondestructive evaluation. Rev. Sci. Instrum. 2009, 80, 025105.

15. Abdulhay, E.; Gumery, P.Y.; Fontecave, J.; Baconnier, P. Cardiogenic oscillations extraction in inductive plethysmography: Ensemble empirical mode decomposition. Conf. Proc. IEEE Eng. Med. Biol. Soc. 2009, 1, 2240-2243.

16. Rheinberger, K.; Steinberger, T.; Unterkofler, K.; Baubin, M.; Klotz, A.; Amann, A. Removal of CPR artifacts from the ventricular fibrillation ECG by adaptive regression on lagged reference signals. IEEE Trans. Biomed. Eng. 2008, 55, 130-137.

17. Husøy, J.H.; Eilevstjønn, J.; Eftestøl, T.; Aase, S.O.; Myklebust, H.; Steen, P.A. Removal of cardiopulmonary resuscitation artifacts from human ECG using an efficient matching pursuit-like algorithm. IEEE Trans. Biomed. Eng. 2002, 49, 1287-1298.

18. Irusta, U.; Ruiz, J.; de Gauna, S.R.; Eftestøl, T.; Kramer-Johansen, J. A least mean-square filter for the estimation of the cardiopulmonary resuscitation artifact based on the frequency of the compressions. IEEE Trans. Biomed. Eng. 2009, 56, 1052-1062.

19. Wu, Y.; Rangayyan, R.M.; Zhou, Y.; Ng, S.C. Filtering electrocardiographic signals using an unbiased and normalized adaptive noise reduction system. Med. Eng. Phys. 2009, 31, 17-26.

20. Lander, P.; Berbari, E.J. Time-frequency plane Wiener filtering of the high-resolution ECG: background and time-frequency representations. IEEE Trans. Biomed. Eng. 1997, 44, 247-2455.

21. Goldberger, A.L.; Amaral, L.A.N.; Glass, L.; Hausdorff, J.M.; Ivanov, P.C.; Mark, R.G.; Mietus, J.E.; Moody, G.B.; Peng, C.K.; Stanley, H.E. PhysioBank, PhysioToolkit, and PhysioNet: components of a new research resourcefor complex physiologic signals. Circulation 2000, 101, E 215-E 220.

22. Friesen, G.M.; Jannett, T.C.; Jadallah, M.A.; Yates, S.L.; Quint, S.R.; Nagle, H.T. A comparison of the noise sensitivity of nine QRS detection algorithms. IEEE Trans. Biomed. Eng. 1990, 37, 85-98.

23. Moody, G.B.; Muldrow, W.E.; Mark, R.G. A noise stress test for arrhythmia detectors. Comput. Cardiol. 1984, 11, 381-384.

24 Chang, K.M.; Liu, S.H. Gaussian Noise Filtering from ECG by Wiener Filter and Ensemble Empirical Mode Decomposition. J. Sign. Process. Syst. 2010, DOI 10.1007/s11265-009-0447-z.

25. Saeed, V.V. Advanced Digital Signal Processing and Noise Reduction, 3rd. ed.; Wiley: New York, NY, USA, 2006.

(C) 2010 by the authors; licensee MDPI, Basel, Switzerland. This article is an Open Access article distributed under the terms and conditions of the Creative Commons Attribution license (http://creativecommons.org/licenses/by/3.0/). 